\title{
Violencias contra las mujeres en el conflicto armado interno colombiano: claves para una lectura crítica.
}

\section{Violence against women in the colombian internal armed conflict: keys for a critical reading.}

"Se sienten morir pero siguen viviendo; manifiestan su impotencia pero siguen pudiendo; dicen que no tienen fuerzas pero sacan fuerzas de donde no las hay" (Ruta Pacífica de las Mujeres, 2013: 60).

Gustavo Macaya-Aguirre* Carolina Stefoni Espinoza**

\section{Resumen}

El presente artículo busca sistematizar y comprender el análisis realizado por la academia y organizaciones de Derechos Humanos sobre la violencia contra las mujeres en el conflicto armado interno colombiano. Para ello, hemos seleccionado y analizado 51 artículos e informes extraídos por palabras clave en diferentes buscadores. Entre los principales hallazgos reportamos que las distintas investigaciones han buscado visibilizar las múltiples formas de violencia que afectan a las mujeres, así como también, indagar sobre los responsables, escenarios, impactos, resistencias e interrelación entre distintas violencias. A partir de lo anterior, planteamos la necesidad de avanzar hacia una analítica de las violencias contra las mujeres como manifestación de poder, abrir la reflexión respecto al vínculo entre violencias y migración internacional forzada y profundizar en la comprensión de los impactos de las violencias que viven las mujeres en su dimensión política.***

Palabras clave: Conflicto armado interno, Colombia, Violencia de género, Violencia sexual, Mujeres.

\footnotetext{
* Estudiante Doctorado en Sociología, Universidad Alberto Hurtado. Facultad de Ciencias Sociales. Santiago, Chile (correo electrónico: gmacaya@uahurtado.cl). Magister en Psicología Social, Universidad Católica del Norte, Antofagasta, Chile. Licenciado en Filosofía y Bachiller en Humanidades, Universidad Alberto Hurtado. Becario ANID-PFCHA/Doctorado Nacional/2020-Folio: 21200311.

* Dra. En Sociología por la Universidad Alberto Hurtado (Chile), Magister en Estudios Culturales, Universidad de Birmingham (UK) y socióloga de la P. Universidad Católica de Chile. Académica Universidad de Tarapacá-Chile. Investigadora Asociada Centro Estudios de Conflicto y Cohesión Social, COES. Dirige actualmente el Proyecto Fondecyt "Rutas y Trayectorias de migrantes venezolanos a lo largo de América del Sur" (№1201130). cstefoni@gmail.com

*** Este manuscrito es parte del proyecto FONDECYT №1201130 financiado por la Agencia Nacional de Investigación y Desarrollo, ANID-Chile
} 


\begin{abstract}
This article seeks to systematize and understand the analysis carried out by the academy and Human Rights organizations on violence against women in the Colombian internal armed conflict. To do this, we have selected and analyzed 51 articles and reports extracted by keywords in different search engines. Among the main findings, we report that the different investigations have sought to make visible the multiple forms of violence that affect women, as well as to inquire about the perpetrators, scenarios, impacts, resistance and interrelationship between different types of violence. Based on the foregoing, we propose the need to move towards an analysis of violence against women as a manifestation of power, open up reflection on the link between violence and forced international migration, and deepen our understanding of the impacts of violence that they experience in their political dimension.
\end{abstract}

Keywords: Colombian internal armed conflict, Colombia, Gender violence, Sexual violence, Women.

Fecha de recepción: Diciembre 2020

Fecha de aprobación: Junio 2021

\title{
Introducción.
}

A lo largo de la historia, las mujeres han sido una y otra vez objeto de violencia durante los conflictos armados y las guerras internas e internacionales. Sin embargo, este tipo de violencia ha sido invisibilizada al punto de sostener que se trataba de una suerte de efecto colateral de la guerra. Es recién a partir de los años noventa -cuando los casos de la antigua Yugoslavia y Ruanda llegan al Tribunal Penal Internacional- que se sostiene que la violencia contra las mujeres en los conflictos armados, en particular la violencia sexual, ha sido utilizada sistemáticamente como una estrategia de guerra. Ello dio inicio a un campo de estudio sobre la violencia de género en los conflictos armados, coincidente con la preocupación de organismos internacionales, en particular el Consejo de Seguridad de Naciones Unidas, que instaló a inicios del siglo XXI la violencia de género como un eje central en su agenda. En este contexto, la violencia de género en el conflicto armado interno colombiano adquirió mayor visibilidad (Fiscó, 2005; Céspedes-Baez, Chaparro \& Stefan, 2014; Chun, S. \& Skjelsbæk, 2010; Meger, 2016; Skjelsbæk, 2001; 2018; Kreft, 2020).

El objetivo del presente artículo es sistematizar y comprender el análisis realizado por la academia y las organizaciones de Derechos Humanos respecto a la violencia contra las mujeres en el conflicto armado interno colombiano. Sostendremos que, si bien existe un análisis respecto de las violencias que viven las mujeres, la identificación de los responsables, los impactos sociales y humanos, así como la relación con otras formas de violencia, subsiste la necesidad de analizar la violencia de género en cuanto 
manifestación de relaciones de poder de carácter más general, acentuando el rol estratégico que ésta tiene en la reproducción de dicha dominación. Por otra parte, planteamos que el análisis del conflicto armado colombiano permite comprender la relación entre violencia y desplazamiento forzado, asi como la necesidad de abordar los impactos de las violencias contra las mujeres no solo en su dimensión subjetiva, sino también comunitaria y política. Esto último permite comprender que las formas de violencia tienen entre sus efectos, la generación de procesos de despolitización y encierro, pero también suscitan la emergencia de agencias y resistencias.

Siguiendo a Segato $(2003,2016)$, comprendemos las violencias contra las mujeres como violencia de género, sostenidas en una estructura de relaciones desiguales que posiciona a las mujeres y lo femenino en un lugar de subordinación respecto de lo masculino, y no puede ser comprendida de manera aislada, individual y ligada casi de manera exclusiva al plano de la intimidad y lo doméstico.

El análisis presentado es resultado de una revisión bibliográfica que incluyó la lectura y fichaje de 51 textos (37 artículos académicos, 9 capítulos de libros y 5 libros) publicados entre los años 1990 y 2020. Esta literatura seleccionada reúne investigaciones cualitativas (14), ensayos o artículos de reflexión teórica (23), estados del arte (6), informes (6) e investigaciones mixtas (2) y fue escogida a partir de la construcción de seis ejes de búsqueda: Violencia de género y migración, violencia de género y conflicto armado, violencia de género en Colombia, violencia sexual y conflicto armado, violencia contra la mujer y conflicto armado y violencia de género contra mujeres afrodescendientes en Colombia, los cuales fueron utilizados en español e inglés.

La presentación de los resultados sigue la siguiente estructura: la primera sección presenta aspectos relevantes en torno a la discusión sobre la violencia de género y sexual en el marco de los conflictos armados, enfatizando cuáles han sido las líneas de interpretación que emergen en este campo. La segunda sección analiza la comprensión de la violencia de género en el conflicto armado interno colombiano, por parte de las distintas investigaciones académicas e informes de Derechos Humanos. La tercera sección, analiza una dimensión que surge de la situación particular del caso colombiano, esto es la relación entre violencia de género y desplazamiento forzado. Finalmente, la cuarta sección plantea algunas reflexiones y preguntas que permiten orientar futuros trabajos en este tema.

\section{Tres ejes analíticos para comprender las formas de violencia contra las mujeres en los conflictos armados}

Las investigaciones que abordan la violencia de género en el marco de conflictos armados internos o de guerras se centran principalmente en la violencia sexual, y tienen como objetivo comprender por qué y cómo ocurren estas violencias, y plantean un marco analítico para su interpretación. Asimismo, ha habido una preocupación sobre el tratamiento que da la justicia, en particular, los dispositivos de justicia transicional y el derecho internacional en materia de Derechos Humanos. Los diversos estudios 
Revista Punto Género N. 15 Junio de 2021

ISSN 0719-0417 / 25- 46

desarrollan reflexiones a partir de las experiencias de diversos países y conflictos armados internos o guerras vividos durante el siglo $\mathrm{XX}$, en particular, toman en sus reflexiones los casos de la antigua Yugoslavia, Ruanda, Sudáfrica, Guatemala, Perú, Colombia, entre otros (Franco, 2007; Boesten, 2010, 2017; Céspedes-Báez, 2010; Céspedes-Báez, et al., 2014; Segato, 2016; Wood, 2016, 2019, Ozcurumez, Akyuz \& Bradby, 2020).

En la revisión de la literatura hemos podido identificar tres líneas interpretativas para comprender el origen de estas violencias en los conflictos armados: i) la violencia de género como un arma de guerra, ii) la violencia de género como un continuum de violencias; y iii) la violencia sexual y la violación como una práctica tolerada. No obstante, es importante recordar que estas interpretaciones se han enfocado principalmente, en el abordaje de la violencia sexual y la violación. A continuación, revisaremos estos tres ejes analíticos.

i) La violencia sexual como arma de guerra

Esta interpretación distingue la agresión sexual en cuanto acto criminal dirigido hacia una persona, de aquella que es utilizada como una estrategia para destruir o dispersar determinados grupos. Autores como Franco (2007) y Meger (2016) plantean que quienes sostienen que la violencia sexual o la violación es un arma de guerra, enfatizan el propósito estratégico de su utilización en los conflictos armados, considerándola como una táctica más dentro de otras para dañar al enemigo. De ahí entonces que, si bien la violación y posterior asesinato de mujeres existe también fuera de los conflictos armados y guerra, en el marco de conflictos armados se trata de algo distinto, tanto por la escala, su nivel de planeación y crueldad.

Esta interpretación se ha convertido en una de las más relevantes para los organismos internacionales y ha permitido que los perpetradores de estas violencias puedan ser juzgados como autores de crímenes de guerra, de manera diferenciada de los delitos comunes. Sin embargo, organismos humanitarios e investigadoras feministas comenzaron a problematizar esta interpretación, planteando que la violencia sexual en los conflictos armados es un fenómeno más complejo que requiere formas de comprensión que no esencialicen ni simplifiquen este fenómeno. En esta línea, investigadoras feministas han criticado el tratamiento dado a la violencia de género y sexual como arma de guerra ligado a temas de seguridad (Chun \& Skjelbæk, 2010; Boesten, 2010, 2017; Meger, 2016; Wood, 2016, 2019; Skjelbæk, 2018; Kreft, 2020). Boesten (2010), a partir del estudio del conflicto armado en Perú, muestra los límites de la interpretación de la violación como un arma de guerra, al señalar que ello oscurece otras formas de violación y violencia sexual durante los conflictos armados generando condiciones de inteligibilidad para que ciertos acontecimientos sean sindicados como violencia política y otros no, a través de ciertos regímenes de violación (rape regimes), con los cuales el cuerpo social e institucional analiza, clasifica y pondera los actos (por ejemplo si se trata de violaciones domésticas o políticas).

No obstante, la invitación de la autora no es a desechar la concepción de la violencia sexual como arma de guerra, sino más bien situar y complejizar su comprensión, 
Revista Punto Género N. 15 Junio de 2021

ISSN 0719-0417 / 25- 46

es decir, distinguir las circunstancias, la relación entre víctima y perpetrador, la motivación y la agencia de la víctima, así como también avanzar hacia una comprensión que no interprete estas violencias como hechos excepcionales (Boesten, 2010, 2017).

ii) Sobre la continuidad de la violencia en tiempos de guerra y paz

Una segunda línea interpretativa sugiere que la violencia contra las mujeres en los conflictos armados y guerras, en especial la violencia sexual, está constituida como un continuum de violencias. Boesten (2017) señala que la noción continuum de la violencia fue desarrollada por la investigadora Liz Kelly en su investigación titulada Surviving Sexual violence (1988). La autora plantea que las distintas experiencias de violencia están conectadas: abuso, intimidación, coerción, trato y fuerza a través de las cuales se busca controlar a las mujeres. Según Boesten, la idea de continuum permite identificar las conexiones que existen entre acciones vistas como criminales, como la violación, y otros actos percibidos y tratados como normales o menores (silbar, coaccionar, acosar). La autora plantea que esta aproximación permite observar cómo determinadas prácticas son ocultadas, institucionalizadas, minimizadas y/o normalizadas en la vida cotidiana, tanto en los tiempos de paz como de guerra y en distintos espacios y relaciones.

En una línea similar, Cockburn (2004) señala que la idea de continuum permite conectar los tipos y ocasiones en los cuales acontece la violencia. Analiza la idea de continuum en tres sentidos. En primer lugar, la violencia de género se experimenta en distintos puntos y niveles de la vida social, como si se viviese en una situación de exposición a las violencias de manera permanente y no exclusivamente durante la guerra. En segundo lugar, la autora plantea que si bien analíticamente es posible distinguir entre pre-conflicto, conflicto, construcción de procesos de paz y reconstrucción, las violencias persisten y transitan a través de distintas formas y en todos los momentos. En tercer lugar, el continuum de las violencias también refiere a su expresión en ámbitos tan distintos como lo económico, político y cultural, superando los límites de la intimidad.

Las posturas críticas a la perspectiva de continuidad también nos permiten observar sus alcances y límites. En primer lugar, Wood (2016) plantea que se trataría de una teoría lineal sobre la violencia sexual, y no permite dar cuenta de la brutalidad de los agentes armados, su masividad y las tecnologías que operan en este proceso. No obstante, reconoce que investigaciones realizadas en distintos lugares de África buscando articular la continuidad de las violencias entre los tiempos de guerra y los periodos de posconflicto, reportaron que en lugares donde las violencias en general, incluida la sexual, habían sido muy intensas, las mujeres tenían una mayor probabilidad de sufrir violencia sexual por parte de su pareja íntima en el periodo posconflicto. Asimismo, reporta que hombres que sufrieron violencia política tenían tasas significativamente más altas de violencia física hacia sus parejas, que aquellos no expuestos a esta violencia, por lo cual habría que pensar la continuidad desde ese sentido.

En segundo lugar, Rita Segato señala que la perspectiva de continuidad genera una indistinción que dificulta la adecuada comprensión de la violencia de género:

"Mi posición no es que en esos bolsones [de población] las formas de la guerra sean una continuidad de la vida doméstica, sino al contrario, que es 
Revista Punto Género N. 15 Junio de 2021

ISSN 0719-0417 / 25- 46

la misma forma de guerra que hace foco en la destrucción del cuerpo de las mujeres y con eso destruye la confianza comunitaria” (Segato, 2016, p. 161).

Esta última observación de Segato, al parecer, no rompe del todo con la tesis del continuum, sino más bien la piensa al revés, es decir, habría una ruptura entre la violencia cotidiana previa y fuera de los marcos de las guerras o conflictos armados; sin embargo, existe una continuidad desde la guerra hacia la violencia que se ejerce en la esfera doméstica y comunitaria en las áreas de conflictos o posterior a estos.

En tercer lugar, Céspedes-Báez, Chaparro y Vargas (2014) señalan que una de las dificultades de sostener la tesis de la continuidad de las violencias es que debilitaría la tesis jurídica de que la violencia sexual es un crimen de lesa humanidad cometida por los actores armados en el marco del conflicto, un aspecto que, en el caso colombiano, distintas organizaciones han buscado remarcar, de cara a la persecución de los perpetradores de estas violencias.

iii) Violencia sexual como práctica tolerada

Wood (2016, 2019) plantea que las aproximaciones anteriores dan cuenta parcialmente de la violencia sexual desarrollada en los conflictos armados al enfocarse en la violación, sobreestimar su presencia y no explicar la ausencia de violación y violencia sexual, por lo que estas no se harían cargo de la variabilidad que presenta este fenómeno. La autora argumenta la existencia de un tipo de violación que no responde a una estrategia o política institucional, y opera más bien como una práctica tolerada, no propiciada por los comandantes a cargo, ni tampoco prohibida de manera efectiva, ni menos castigada y que puede emerger como una forma de cohesión al interior de grupos donde se ha utilizado el reclutamiento forzado, como una forma de autopago e, incluso, como formas de afirmación de la masculinidad y reclamo de cierto estatus. Por su parte, los comandantes no siempre pueden ni están dispuestos a castigar a los combatientes y terminan aceptando las distintas prácticas de violación y violencia sexual ejercidas por estos.

En este marco comprensivo, para saber frente a qué tipo de violaciones estamos, es importante saber si se castiga o no a quien realiza estos actos. Si está prohibida, pero no se castiga, entonces es una práctica tolerada, y si está prohibida y se castiga a quien la realiza, no es una política organizacional. Además, si se presenta a pesar de los castigos, es una práctica muy arraigada. Por último, si hay evidencia de que la violación u otro acto de violencia sexual fue ordenado, entonces estamos frente a una violencia sexual como política organizacional (Wood, 2019).

De ahí entonces, para Wood (2019) en los conflictos armados es posible encontrar la violación como política institucional, que puede incluir la violación como estrategia de guerra, la violación como práctica tolerada y también ausencia de violación. Según la autora, la distinción entre la violación como práctica y como política permite comprender mejor la variabilidad de los patrones de violación, así como también puede contribuir a la formulación de políticas públicas adecuadas, prestando atención a las distintas dinámicas que puedan darse al interior de los conflictos armados. 
Revista Punto Género N.15 Junio de 2021

ISSN 0719-0417 / 25- 46

Ciertamente, cada una de estas aproximaciones aporta con énfasis distintos en la comprensión de estas experiencias y sin duda están presentes en distintas investigaciones que abordan las violencias vividas por las mujeres en los conflictos armados. En el caso colombiano, la violencia como arma de guerra y como un continuum han tenido una predominancia para su comprensión; sin embargo, el campo de estudio sobre la violencia hacia las mujeres en Colombia, en particular la sexual, es amplio y ha profundizado en distintos aspectos de esta, cuestión que revisaremos a continuación.

\section{Dimensiones de las violencias contra las mujeres en el conflicto armado interno colombiano}

A continuación se abordan distintos aspectos relacionados a las maneras en cómo se ha comprendido la violencia contra las mujeres en el marco del conflicto armado interno colombiano. Abordaremos i) la sistematización de las principales formas de violencia identificadas y reportadas por las investigaciones y cómo estas han sido interpretadas, ii) quiénes han sido los principales perpetradores de estas violencias y por qué muchas de estas situaciones no se denuncian, y iii) los impactos de estas violencias en la vida de las mujeres que las han sufrido.

i) Violencia de género y su interpretación

El conflicto armado interno colombiano lleva más de 70 años de historia. Distintos autores marcan como punto de inicio el asesinato del político Jorge Eliécer Gaitán en 1948. Ciertamente, este conflicto ha tenido múltiples etapas y no se ha desarrollado de manera homogénea todo el tiempo ni en todos los territorios del Estado colombiano. En las últimas tres décadas del siglo $\mathrm{XX}$, se comenzó a articular una nueva fase en esta guerra, caracterizada por la lucha por el control territorial por parte de los distintos grupos armados: estatales, contraestatales (guerrillas) y paraestatales (paramilitares), quienes además están atravesados por la acción de un cuarto actor: el narcotráfico.

Las afectaciones del conflicto armado sobre la población civil han sido múltiples y, "en muchos casos, el terror ha penetrado las rutinas, no solo del espacio público y de la vida colectiva de la comunidad, sino del ámbito cotidiano e íntimo de la familia, el amor y la atracción sexual" (Meertens, 2000,p 120).

Las distintas investigaciones e informes dan cuenta de que la violencia contra las mujeres, y en particular la violencia sexual y la violación, se dan en distintos escenarios vinculados directa o indirectamente a la guerra. Así, es posible identificar, al menos, cinco escenarios en el que estas violencias se agudizan, a saber: el escenario de disputa territorial, escenario donde un determinado actor armado ejerce presencia y control en el territorio; al interior de los grupos armados guerrilleros y paramilitares; durante el desplazamiento forzado $\mathrm{y}$, finalmente, en escenarios intracomunitarios, intrafamiliar o de relaciones de pareja (Amador Ospina, 2017; Centro Nacional de Memoria Histórica de Colombia [CNMH], 2017).

Los regímenes de violación o de violencia sexual (Boesten, 2010) han hecho visibles y ponderables, como ligados al conflicto, aquellos cometidos por los actores 
Revista Punto Género N. 15 Junio de 2021

ISSN 0719-0417 / 25- 46

armados, visibilizando parcialmente o silenciando aquellos cometidos durante los procesos de desplazamiento forzado, escenarios intracomunitarios, intrafamiliar o los que se dan al interior de los grupos armados (Amador Ospina, 2017).

El informe desarrollado por la Ruta Pacífica de las Mujeres (2013), da cuenta de que las mujeres han sufrido múltiples formas de violencia física, psicológica y sexual en el conflicto armado interno, tales como hostigamientos y destrucción de bienes (requisas, allanamientos, amenazas, seguimientos y vigilancia), tortura física, psicológica y sexual, violaciones a la libertad personal (detenciones arbitrarias, reclutamiento forzado, toma de rehenes, confinamiento), desplazamiento forzado interno, individual, familiar o colectivo y violaciones al derecho a la vida (ejecuciones extrajudiciales, desapariciones forzadas, atentado y heridas).

Situación similar es la reportada por Fiscó (2005), quien realizó un análisis de distintos actos de violencia sufrida por mujeres, en las cuales encontró ocho categorías que remiten a distintos tipos de violencia: Acceso carnal violento, actos sexuales violentos, prostitución forzada/esclavitud sexual, chantajes sexuales, esterilización forzada y aborto forzado, "reglas de convivencia" impuestas que implican discriminación y violencia de género, señalamientos que desencadenan violencia directa sobre la mujer por presuntas relaciones afectivas con algún actor del conflicto armado, violencia intrafamiliar contra la mujer que se agudiza por efecto del conflicto armado interno. De todos ellos, los hechos victimizantes con prevalencia fueron aquellos que están dentro de la categoría acceso carnal violento ${ }^{1}$.

Si bien todas las mujeres que viven en zonas donde se desarrolla el conflicto armado se encontrarían potencialmente en peligro de sufrir algún tipo de violencia, son las mujeres lideresas quienes están expuestas, en mayor medida, a ser blanco de distintas formas de violencia por parte de los actores armados, quienes pujan por evitar que las mujeres se organicen, capaciten y exijan sus derechos, pues ellas obstaculizan el avance y control territorial de los grupos armados (Defensoría del Pueblo, 2011).

Las investigaciones revisadas sobre violencia hacia mujeres afrodescendientes del Pacífico colombiano, especialmente de Buenaventura, dan cuenta del ensañamiento con el cual son asesinadas, sobre todo las mujeres jóvenes, en crímenes que son abordados como privados, percibidos como vinculados a relaciones de pareja íntima y atribuidos a una comprensión de la población afrodescendiente como personas culturalmente violentas, ocultando que, detrás de estos hechos, está el conflicto armado interno y la presencia de actores armados en los territorios (Benjumea \& Loaiza, 2018; Defensoría del Pueblo, 2011; Lozano, 2016).

En este sentido, la Defensoría del Pueblo (2011) plantea que los homicidios han cumplido dos propósitos, a saber: castigar y aleccionar. Generalmente, estos asesinatos

\footnotetext{
${ }^{1}$ La autora toma el concepto acceso carnal violento del Código Penal Colombiano, el cual, en su artículo 212 señala "Se entenderá por acceso carnal la penetración del miembro viril por la vía anal, vaginal u oral, así como la penetración vaginal o anal de cualquier parte del cuerpo humano u otro objeto". También toma el artículo 138, sobre las penas a quienes cometan este delito a personas protegidas en el marco del conflicto armado.
} 
Revista Punto Género N. 15 Junio de 2021

ISSN 0719-0417 / 25- 46

también van acompañados de otros signos de violencia, como tortura, violación y desmembramiento, y reflejan un particular ensañamiento en su ejecución.

Estas múltiples formas de violencia hacia las mujeres son interpretadas por las distintas investigaciones como el despliegue de acciones que operan como estrategia de guerra para la desestabilización del enemigo, la ruptura de las comunidades, la aniquilación de los liderazgos comunitarios, como forma de atemorizar a la población civil y ejercicio del control territorial a través del cuerpo de las mujeres (Amnistía Internacional, 2004; Fiscó, 2005; Ruta Pacífica de las Mujeres, 2013; Segato, 2016; Wilches, 2010; CNMH, 2017; Benjumea \& Loaiza, 2018; Salcedo \& Paes-Machado, 2019). De la misma manera, Kreft (2020) señala que, mediante la violación, el hombre consolida su posición al interior del grupo armado, lo que permite comprender la violencia sexual en el marco del conflicto armado como un ejercicio del poder masculino.

En la constitución de las violencias experimentadas en específico por las mujeres Afrodescendientes, Marciales plantea la importancia de considerar las construcciones históricas y jerárquicas de género y raza desde la colonia hasta la actualidad, las que no solo han posicionado a estas mujeres en una condición de subalternidad respecto a otras mujeres y el resto de la sociedad, sino también, han producido relaciones marcadas por una racialización y sexualización de sus cuerpos, por lo cual, estas violencias, "además de considerarse como una expresión de violencia de género, debe ser analizada como una clara manifestación de racismo estructural" (Marciales, 2015, p.86).

Distintas investigaciones (Arroyo \& Álvarez, 2018; Kreft, 2020), en sintonía con lo planteado por investigadoras feministas, sugieren que la violencia de género asociada al conflicto está sostenida en estructuras patriarcales muy arraigadas en la sociedad colombiana. El conflicto armado habría exacerbado formas de violencia ya instaladas, aunque reconocen que las violencias vividas en el marco del conflicto armado interno tienen ciertas diferencias y especificidades en sus manifestaciones, fines y alcances.

ii) Sobre los responsables y los silencios.

Las investigaciones revisadas señalan que las violencias son perpetradas por miembros de todos los grupos armados, legales e ilegales, sin embargo, también advierten las dificultades de las mujeres para identificar con claridad quiénes son los responsables de estos actos (Amnistía Internacional, 2004; Defensoría del Pueblo, 2011; CNMH, 2017; La Ruta Pacífica de las Mujeres, 2013; Benjumea \& Loaiza, 2018; Marciales, 2018). El informe de La Ruta Pacífica de las Mujeres señala: "las respuestas, casi siempre, muestran dudas, inseguridad, contradicciones, que expresan tanto el miedo frente a los perpetradores como a la violencia cruzada de que han sido objeto" (2013,p. 199).

Una de las razones principales por las cuales las mujeres no denuncian las agresiones sexuales sufridas por parte de los actores armados es el miedo a las represalias de los perpetradores, en particular, al asesinato de ellas mismas o a miembros de su familia. Ese miedo no solo es real en la experiencia subjetiva de la mujer sobreviviente que lo experimenta, sino también es una posibilidad fáctica en el marco del conflicto armado. Asimismo, el miedo al rechazo y la culpa, también inhibe a las mujeres a 
Revista Punto Género N. 15 Junio de 2021

ISSN 0719-0417 / 25- 46

denunciar estos hechos (Fiscó, 2005; Benjumea \& Loaiza, 2018; Lucumí, 2012, Defensoría del Pueblo, 2011; Carrillo, 2014).

Los factores antes mencionados disminuyen la probabilidad de que una persona pueda acceder a la red de protección para víctimas de la violencia. Frente a estas dificulades, las organizaciones no gubernamentales cumplen un rol central en la atención a las mujeres víctimas de violencia, sobre todo sexual, pues entregan una primera respuesta de salud y acompañamiento en el proceso de reparación (Urrego-Mendoza, 2007; Ruta Pacífica de las Mujeres, 2013; Orduz, 2015; Benjumea \& Loaiza, 2018; Peñaranda \& Sulewski, 2017; Carrillo, 2014; Arroyo \& Álvarez, 2018).

iii) Los impactos de estas violencias y la emergencia de formas de resistencia.

Respecto de los impactos de estas violencias en la vida de las mujeres, el informe de la Comisión de la Verdad de La Ruta Pacífica de las Mujeres (2013) señala que están vinculados a impactos socio afectivos y proyecto de vida (condiciones afectivas, económicas, proyectos de vida truncados, deterioro de las condiciones de vida y repercusiones en su vida pública y privada), impactos específicos a ellas como mujeres (estigmatización social, identidad como mujeres, sexualidad y separación familiar, abandono) y consecuencias en la salud y el cuerpo (hospitalizaciones, discapacidad física o sensorial, heridas, fracturas, dolores crónicos, adicciones, enfermedades).

Asimismo, Benjumea y Loaiza (2018) reportan una serie de impactos causados por la violencia sexual y, en particular por la violación, los que son recogidos en su trabajo con mujeres afrocolombianas de Tumaco, Nariño, y pudiesen resultar verosímiles también en otros grupos de mujeres. Los impactos físicos van desde infecciones urinarias, ITS, hemorragias hasta embarazos y/o abortos forzados. Otros tipos de consecuencias están en los trastornos de alimentación y sueño, tales como dificultad para dormir, desvelo, pesadillas, insomnio. Algunas de ellas comienzan a padecer dolores constantes en sus cuerpos, muchos de ellos con una fuerte vinculación con la emocionalidad y difíciles de diagnosticar por médicos como ligados a una enfermedad concreta.

Los impactos de estas violencias a nivel psicológico y emocional son muy potentes; a los sentimientos de miedo, terror, culpa y soledad, se suma también la rabia, los intentos suicidas, los comportamientos agresivos, la desconfianza hacia los otros, particularmente los hombres, la merma en la confianza hacia ellas mismas, entre otras, lo cual dificulta la posibilidad de armar y rearmar sus proyectos de vida (Lucumí, 2012).

Benjumea y Loaiza (2018) reportan que el sentimiento que embarga la vida de estas mujeres puede ser catalogado como una tristeza profunda, emergiendo como "una respuesta normal y previsible ante hechos de violencia tan atroces como los sexuales" (p.66). Asociado a lo anterior, las autoras señalan que las mujeres víctimas de violencia sexual también experimentan culpa y vergüenza por las experiencias vividas y, en particular, por los constantes señalamientos que suelen acompañarlas cuando se deciden a contar y denunciar la situación, lo cual agudiza la experiencia de dichos sentimientos.

Otro ámbito de daños se vincula con su vida sexual, dado que ellas comienzan a tener una serie de dificultades en términos del placer y disfrute ligado al sexo. Las autoras 
reportan que algunas mujeres vieron muy mermadas sus relaciones de pareja con posterioridad a los hechos, al punto que esto implicó el fin de las relaciones con sus compañeros, quienes las juzgaban como responsables de los hechos o, en el caso de no conocer el motivo detrás de la evasión o negativa de ellas, las acusaban de infidelidad (Benjumea \& Loaiza, 2018).

Es importante considerar que las experiencias de violencia también laceran las practicas culturales y comunitarias propias de esas comunidades negras del Pacífico, pues muchas de las actividades realizadas colectivamente de manera cotidiana se dejan de realizar por temor a represalias, inseguridad y desconfianza (Carrillo, 2014; Goldscheid, 2020). Por lo tanto, los daños de estas violencias no son solamente individuales y familiares, también son comunitarios, pues "estas violencias logran su propósito con hechos que desarticulan a las comunidades, que socaban sus tradiciones de solidaridad y ayuda mutua" (Lozano, 2016: 15).

Una pregunta importante frente a estas afectaciones e impactos tan potentes a nivel físico, psicológico, emocional y relacional, dice relación con los procesos de recuperación, sanación y resistencia. En esa línea, es relevante consignar la importancia de la medicina ancestral, el uso de hierbas y remedios caseros para poder aliviar los dolores y malestares posteriores al ataque sexual vivido (Benjumea \& Loaiza, 2018).

Benjumea \& Loaiza (2018) señalan que los talleres y encuentros promovidos por organizaciones de la sociedad civil, si bien son espacios de encuentro, contención y resistencia para las mujeres que deciden compartir sus experiencias, no solo favorecen la escucha y sanación, sino también devienen en procesos organizativos de las propias mujeres y la emergencia de liderazgos que actúan como resistencia a las prácticas de opresión cotidiana que imponen los grupos armados. Estos espacios permiten iniciar un camino de reconstrucción y recomposición emocional a través de la comunicación de la vivencia de manera amplia lo cual posibilitaría, a su vez, generar la recomposición política, la acción colectiva y de proyecto de vida (Meertens, 2000; Jimeno, 2007; González Gil, 2012; Salcedo \& Paes-Machado, 2019).

La experiencia común de las mujeres sobrevivientes que narran estas violencias es el quebranto provocado por los hechos victimizantes, experiencia que está atravesada por contraposiciones: "se sienten morir pero siguen viviendo; manifiestan su impotencia pero siguen pudiendo; dicen que no tienen fuerzas pero sacan fuerzas de donde no las hay" (Ruta Pacífica de las Mujeres, 2013, p. 60). Esta fuerza que mueve a las sobrevivientes, a pesar de los hechos de violencia, se expresa en tres ámbitos de acción, a saber: resistir y movilizarse en nombre de los vínculos, rehacer las condiciones de humanidad y tejer la vida colectiva (González Gil, 2012; Ruta Pacífica de las Mujeres, 2013; Carrillo, 2014; Lozano, 2016; Peñaranda y Sulewski, 2017; Cruz, Calderón, Flores \& Cardozo, 2018).

A continuación, abordaremos los vínculos entre la violencia que viven las mujeres y el desplazamiento forzado, que se constituye también como una manera de responder ante las distintas formas de violencia presentes en los territorios y que afectan a las mujeres de manera particular. 
Revista Punto Género N. 15 Junio de 2021

ISSN 0719-0417 / 25- 46

\section{Violencia contra las mujeres y desplazamiento forzado en el conflicto armado colombiano}

El Alto Comisionado de Naciones Unidas para los Refugiados [ACNUR] (2020) estimó que, entre los años 2010 y 2019, más de 79 millones de personas en el mundo se desplazaron forzadamente al interior de sus países, siendo Colombia el país con mayor número de desplazamiento forzado interno.

Donny Meertens (1999: 32) señala que en los años 1988 y 1989, en los que comienza a aumentar la violencia del conflicto armado, empiezan a darse con mayor magnitud distintos procesos de desplazamiento forzado en Colombia. Al año 1994, la Conferencia Episcopal de Colombia reportó alrededor de 600.000 desplazados, concentrados en ciertas zonas del país como Urabá, Córdoba, Magdalena Medio, los Orientales, Arauca y Cauca/Putumayo. Actualmente, según el reporte del Registro Único de Víctimas, al 30 de junio de 2020, habían 8.047.756 personas registradas como víctimas de desplazamiento forzado interno con motivo del conflicto armado interno, de ellas, 4.146.835 eran mujeres (Unidad para la atención y reparación integral a las víctimas [Colombia], 2020).

Para Ruiz (2011), el desplazamiento forzado interno por muchos años se caracterizó por el tránsito desde zonas rurales y semi rurales hacia distintos centros urbanos del país, y no se explica únicamente por la presencia de actores armados en la zona, lo que sin duda es un elemento importante. Resulta central atender que son los distintos hechos de violencia recayendo sobre la población civil que habita estos territorios, los que generan desplazamiento forzado interno, acompañados de una profundización en las condiciones de pobreza, dada la expropiación de la tierra, con la consecuente pérdida de propiedad privada, el quiebre en el tejido social y la perdidas de las fuentes de ingresos. Pese a ello, distintos autores han señalado que el desplazamiento forzado interno también es un escenario que permite la reconstrucción de la vida de las personas, genera cambio en los roles tradicionales de género, proyectos de vida y posibilita la generación de espacios de acción colectiva (Meertens, 1999, 2000; Segura \& Meertens, 1997; González Gil, 2012; Pareja Amado \& lañez Domínguez, 2014).

Respecto al desplazamiento forzado de las mujeres, Céspedes-Báez (2010) plantea que el concepto de desproporción permite comprender cómo el desplazamiento forzado genera impactos diferenciados para la vida de estas mujeres y sus derechos humanos, debido a la intensidad y las experiencias que viven antes, durante y después del desplazamiento, donde muchas de ellas "quedan expuestas en más alto grado (...) a la explotación doméstica, la informalidad laboral, la desprotección de su derecho a la salud, la dificultad en el acceso a la justicia y, por supuesto, la violencia sexual" (p.285).

La revisión de la literatura permite identificar cinco dimensiones a través de las cuales se vincula la violencia de género con el desplazamiento forzado en el conflicto armado. En primer lugar, encontramos la relación entre tipos de victimización y desplazamiento forzado. Salcedo y Paes-Machado (2019) identifican dos tipos de 
Revista Punto Género N. 15 Junio de 2021

ISSN 0719-0417 / 25- 46

victimización que gatillan procesos de desplazamiento forzado de mujeres. Por una parte, una victimización directa, es decir, hechos violentos dirigidos hacia las propias mujeres (violencia sexual o pérdida de bienes materiales) y, por otro, una victimización indirecta relacionada con hechos ocurridos a personas cercanas a su entorno, pero que terminan afectándolas a ellas directamente (asesinatos, desapariciones forzadas, resistencia al reclutamiento forzado de los hijos). Por ejemplo, la búsqueda de familiares desaparecidos (esposos, hermanos, hijos, entre otros), devienen en amenazas contra sus propias vidas cuando los grupos armados dan una instrucción clara respecto de que deben dejar de buscar e irse a otro lugar (Pareja Amado e lañez Domínguez, 2014).

Una segunda dimensión enfatiza la relación entre violencia sexual, el despojo de tierras y el desplazamiento forzado. Céspedes-Baez (2010) ha planteado, a partir de la evidencia recogida por distintos organismos, que la violencia sexual sufrida por las mujeres en el conflicto armado interno ha tenido distintos fines, y los testimonios de las víctimas evidencian la relación entre violencia sexual, desplazamiento forzado y despojo de tierras.

La comprensión de la relación entre las distintas formas de violencia permite salir de una noción que tiende a reducir la violencia sexual a una mera práctica de consumo masculino de los cuerpos femeninos y visibilizar que, a través de ella, se buscan también otros objetivos estratégicos ligados con los móviles propios de los grupos armados y su acción en los territorios, en un marco de desterritorialización de la guerra en Colombia. En este caso, la tesis general de la autora es que, mediante la violencia sexual y el desplazamiento forzado interno, los actores armados pueden tomar posesión de la tierra (Céspedes-Baez, 2010).

Una tercera dimensión plantea que las mujeres que experimentan abusos y violencia sexual no son necesariamente acogidas, sino muchas veces son rechazadas y negadas por sus familias y comunidades, al punto de la expulsión. En otras palabras, las mujeres deben desplazarse forzadamente debido a los impactos de los hechos de violencia en las relaciones con su familia y comunidad (Salcedo \& Paes-Machado, 2019).

La cuarta dimensión relaciona el desplazamiento forzado de niñas y adolescentes al acoso sexual que experimentan por parte de miembros de los grupos armados (paramilitares o miembros de las FARC), quienes las persiguen para tener algún tipo de relación con ellas, incluido el reclutamiento forzado, principalmente con fines domésticos o sexuales. En este contexto, las mujeres que las cuidan (generalmente sus madres) deben tomar la decisión de desplazarse, como una forma de prevención y para arrebatarle sus hijas a la guerra (Fiscó, 2005; Wirtz et al., 2014; Pareja Amado \& lañez Domínguez, 2014, Echeverri, 2016).

Finalmente, la última dimensión dice relación con los señalamientos y amenazas contra algunas personas, ya sea debido a deudas pendientes con el grupo armado, ejercer algún tipo de liderazgo comunitario, ser señaladas como colaboradoras o estar involucradas sentimentalmente con algún miembro del grupo enemigo. Estas situaciones también generan procesos de desplazamiento forzado (Meertens, 2000; Wirtz et al., 2014). 
Revista Punto Género N. 15 Junio de 2021

ISSN 0719-0417 / 25- 46

Salcedo y Paes-Machado (2019) en su investigación distinguen tres modalidades de desplazamientos forzado: campo-campo, campo-ciudad y pendular, las cuales hay que interpretar a la luz de los escenarios y motivos que llevan al desplazamiento. El desplazamiento campo-campo se caracteriza por producirse de manera intempestiva y poco organizada, así como también por "la salida de los lugares de origen hacia las cabeceras de las poblaciones cercanas y se hace bajo la presión de los combates entre grupos armados o las incursiones sorpresiva de estas en las poblaciones" (p.114). En general, se trata de desplazamientos breves, espontáneos y colectivos y se dan por el recrudecimiento de la violencia en un momento determinado y una vez que desciende el nivel de violencia, vuelven a sus lugares de origen.

Por su parte, el desplazamiento forzado campo-ciudad: "se hace de forma dispersa y planeada obedeciendo a las circunstancias propias del ambiente de zozobra y terror instaurado por los grupos armados" (Salcedo y Paes-Machado, 2019, p. 115). En este sentido, se trata de "un tipo de movimiento preventivo y en algunos casos silencioso ya que implica solo a un núcleo familiar o madres jefas de hogar con sus hijos" (p.115).

La tercera modalidad es el desplazamiento forzado pendular, en donde se produce una primera salida para protegerse de un peligro y, luego, retornar para recuperar algo de lo abandonado y, a partir de ello, se vuelve a desplazar a otro lugar.

Carrillo (2014) agrega un elemento central al señalar que las modalidades del desplazamiento forzado interno están atravesadas por los recursos económicos a los que puedan acceder las mujeres y sus familias cuando se ven en la obligación de desplazarse, así como también por las redes que puedan tener en otros centros urbanos. Las mujeres con menos recursos se desplazan entre los barrios, mientras que aquellas con acceso a algo más de dinero lo hacen hacia otros centros urbanos, donde tienen personas conocidas que las pueden acoger inicialmente.

Otro asunto importante dice relación con que las experiencias de violencia no acaban con el desplazamiento forzado interno, no desaparecen con la huida de las comunidades asediadas por el conflicto armado, sino también se ven expuestas a éstas durante el proceso de desplazamiento y la llegada a un nuevo lugar. Sin embargo, los perpetradores cambian, pueden ser extraños, los dueños de los lugares a donde llegan, sus compañeros sentimentales o familiares, o los actores armados que predominan en el lugar de llegada (Wirtz et al., 2014; Montoya, Romero \& Jerez, 2013).

Finalmente, existen algunas investigaciones que dan cuenta de los cambios y transformaciones experimentados por las mujeres una vez que se han instalado en el lugar de llegada. Junto con el empobrecimiento, desarraigo y violencia que ello supone, Pareja Amado e lañez Domínguez (2014) plantean que las principales dificultades están ligadas a su baja calificación e instrucción escolar, que les impide acceder a trabajos formales. Asimismo, los saberes que ellas tienen producto de las actividades desarrolladas en el campo se ven desvalorizados en un ambiente urbano. Algunas mujeres logran ingresar a programas de formación y empleabilidad e, inclusive, algunas montan su propio emprendimiento, cuestión que no siempre llega a buen término, dada la inexistencia de un acompañamiento adecuado o inviabilidad del rubro elegido. Otras se 
incorporan al empleo informal doméstico y logran percibir ingresos, aunque bajo condiciones de trabajo precario. Las estrategias utilizadas para enfrentar los escenarios puestos por el desplazamiento forzado, van transformando y redefiniendo las relaciones y roles de género, facilitada por la inserción de las mujeres en el trabajo formal e informal en el medio urbano, lo que las constituye, en muchos casos, en jefas de sus hogares (Meertens, 1999, 2000).

Tal como lo señala el informe de La Ruta Pacífica de las Mujeres (2013), a pesar de la vulneración de derechos y los traumáticos acontecimientos de violencia que las despojaron de sus medios de vida y sus comunidades, una parte de estas mujeres se han incorporado a procesos organizativos comunitarios y/o de mujeres, entretejiendo nuevamente relaciones, que abren paso a la generación de un cambio en sus vidas y les permite experimentar apoyo de otras mujeres. En esa misma línea, González Gil (2012) señala que la vida en los lugares a los que llegan esas mujeres y sus familias implican y exigen procesos de organización y vinculación para satisfacer distintas necesidades que emergen durante el periodo de llegada. Estas acciones suponen de las personas una cierta identificación sobre problemas comunes que les afectan y la generación de consensos, expresión de dinámicas de acción colectiva y pública, donde la participación de las mujeres ha sido fundamental.

No obstante, no todas las mujeres desplazadas generan redes de asociación, ni tampoco dan a conocer su situación de desplazadas, no solo como una estrategia de invisibilización ante posibles riesgos, sino también, por miedo al estigma de ser consideradas guerrilleras, paramilitares o colaboradoras de estos grupos por parte de los habitantes que residen en las ciudades a las cuales llegan y con quienes se relacionan (Pareja Amado e lañez Domínguez, 2014; Arroyo \& Álvarez, 2018).

\section{Reflexiones emergentes a partir de la revisión}

La literatura respecto de las distintas violencias que viven las mujeres colombianas en el marco del conflicto armado interno muestra la diversidad de estas y da cuenta de cómo pueden ser comprendidas como una exacerbación de experiencias de violencia que las mujeres viven por fuera y con anterioridad al conflicto armado. A continuación, nos referiremos a dos elementos que orientan nuestras reflexiones finales.

i) Hacia una analítica de las violencias como manifestación de poder

Siguiendo a Amador Ospina (2017), sostenemos que la interpretación que realizan los distintos estudios revisados sobre violencia de género, en particular la sexual en Colombia, muy a menudo tienden a utilizar categorías analíticas no necesariamente fundamentadas en estudios empíricos, sino que hacen eco de grandes "eslóganes" que presentan estas violencias como arma de guerra, estrategia de guerra, control territorial, continuum de violencias. Ello invisibiliza los mecanismos a través de los cuales operan las diferentes formas de expresión de violencia sexual, quedando ocultos o silenciados detrás de estas grandes etiquetas. En este sentido, pensamos que las claves analíticas proporcionadas por Segato (2016), pueden contribuir a dar una mayor, mejor y más 
Revista Punto Género N. 15 Junio de 2021

ISSN 0719-0417 / 25- 46

profunda comprensión e inteligibilidad a estas violencias, cuando señala que, en estos crímenes es importante interrogarlos de múltiples maneras: el lugar en el que ocurren, sus propósitos, significados y condiciones de posibilidad; ¿Quién los realiza? ¿Dónde? ¿Cuándo? ¿A quién le habla? ¿Cuál es el mensaje que se busca transmitir?

En esta línea, si las violencias que viven las mujeres en general y la violencia sexual y la violación en particular, se inscriben no en el ámbito de satisfacción del deseo sexual, sino en la esfera de las relaciones poder (Segato, 2003, 2016), entonces, aquello a lo que deberían apuntar los análisis es a una analítica de la violencia de género como manifestación de relaciones de poder (Foucault, 2012).

Siguiendo a Foucault (2012), pensar estas distintas formas de violencias desde una analítica de las relaciones de poder, implica interrogarse más cómo opera, que qué es, también requiere ser pensada y abordada por fuera de los marcos que impone el derecho, la ley. Asimismo, requiere interrogarse con profundidad qué efectos se buscan producir con estas acciones, ¿qué se busca producir por medio de la violencia por parte de quienes la ejercen? ¿Cuáles son los efectos que busca producir y cuáles efectivamente termina generando?

Pensamos que, siguiendo a Boesten (2010), es relevante preguntarse qué ha quedado oculto, silenciado, parcial o totalmente invisibilizado en estos regímenes de comprensión de la violencia contra las mujeres en el ámbito del conflicto armado. Amador Ospina (2017) parece estar en la dirección correcta cuando señala que las investigaciones en el campo de la violencia de género, y en particular la violencia sexual, históricamente se han movido en una invisibilidad estructural, sin embargo, aquello que la lleva a ser visible, de igual forma, la aborda de manera parcial, donde siguen quedando elementos que no terminan de aparecer del todo.

En ese sentido, la literatura analizada, al enfocarse principalmente en tratar de comprender las violencias que afectan a las mujeres en los conflictos armados, han centrado su atención principalmente en la violencia sexual perpetrada por los actores armados a fin de generar un marco de inteligibilidad para permitir su comprensión dentro de las distintas formas de opresión y violencias que viven las mujeres dentro y fuera de las zonas o tiempos de guerra y conflicto, implicando que otras formas de violencia que ocurren en otros espacios tengan una visibilización parcial o hayan quedado silenciadas.

Debido a ello, consideramos relevante seguir abriendo el campo y profundizar en la problematización y comprensión de las violencias de género que afectan a la población LGBTI, mujeres indígenas y afrodescendiente y seguir pensando y articulando las violencias intracomunitarias, intrafamiliares y al interior de las parejas, en un campo que las vincule con aquello que acontece en los conflictos armados. Es pensar la continuidad de las violencias, tal como lo sugiere Segato (2016), no tanto desde la casa a la guerra, sino más bien, desde la guerra hacia las comunidades, familias y relaciones de pareja.

De la misma forma, es importante que las investigaciones consideren y tengan siempre presente que las violencias de género son actos con implicancias políticas profundas, y sus efectos a nivel comunitario, organizativo, familiar e individual tienen relación con el apartamiento y separación de estas personas de lo público, a través del 
miedo y el terror producidas por las distintas formas de violencia física, verbal y sexual a la que se ven expuestas. Por tanto, la violencia de género en general, y la sexual en particular, no son un hecho privado, individual, íntimo y doméstico, aun cuando estas emerjan en el seno de la intimidad del espacio privado, pues sus efectos están vinculados a lo público, a la posibilidad de ejercer plenamente los derechos como ciudadanas y poder desarrollar el proyecto individual o colectivo que las personas deseen.

ii) Violencias, desplazamiento forzado y migración internacional forzada

Es importante insistir en que el desplazamiento forzado habitualmente es antecedido por otro hecho que las llevó a tomar la decisión de salir forzadamente del territorio donde habitaban. Esta sutileza es clave, ya que en general las personas terminan siendo rotuladas como "desplazadas", invisibilizando las experiencias anteriores de violencia.

Otro aspecto menos abordado es la conexión entre desplazamiento forzado internacional y violencia de género, como si los análisis de los impactos del conflicto armado en la vida de las mujeres quedaran circunscritos a los límites del Estado-nación y hubiese una suspensión de las experiencias más allá de las fronteras. Esto da cuenta de distintas formas de nacionalismo metodológico (Wimmer \& Glick Schiller, 2003) que necesitamos romper, y en el cual los estudios migratorios pueden contribuir a pensar la continuidad de estas experiencias y cómo las afectaciones de las violencias del conflicto permanecen y se transforman a lo largo de la trayectoria migratoria (Ozcurumez et al., 2020).

En esa línea, pensamos en tres desafíos relevantes para la investigación: primero, articular y comprender el vínculo entre las experiencias de violencia de género acontecidas en el marco del conflicto armado, los procesos de desplazamiento forzado interno y la migración internacional forzada transnacional. El segundo dice relación con el conocimiento y análisis de otras experiencias de violencias vividas a lo largo de su trayectoria migratoria y, en tercer lugar, comprender los procesos de construcción y reconstrucción de las identidades, subjetividades y resistencias desarrolladas por estas mujeres en sus experiencias migratorias.

Esto es clave de cara a pensar las relaciones entre migración internacional forzada, género y violencias, cuestión que supone la pregunta de si la migración internacional, el exilio, la huida del país, implica efectivamente el fin de las amenazas y las violencias para estas personas y cuáles son las circunstancias y condiciones de posibilidad que generan la reproducción constante de esas violencias y/o los cambios o transformaciones posibles de evidenciar.

De la misma manera, estos desafíos han de interpelar las formas en cómo habitualmente se interpreta el desplazamiento forzado o la migración transnacional forzada, desde una dimensión más bien negativa, para avanzar hacia una comprensión que da cuenta cómo estas experiencias también son formas de resistencia frente a la opresión y violencia cotidiana que viven las mujeres en el territorio en el que habitan, convirtiéndose en una manera de decir, una y otra vez, sí a la vida. 
Revista Punto Género N.15 Junio de 2021

ISSN 0719-0417 / 25- 46

\section{Bibliografía}

Alto Comisionado de Naciones Unidas para los Refugiados [ACNUR] (2020): Tendencias globales desplazamiento forzado en 2019. Disponible en: https://www.acnur.org/stats/globaltrends/5eeaf5664/tendencias-globales-dedesplazamiento-forzado-en-2019.html

Amador Ospina, Marcela (2017): "Interpelando la invisibilidad estructural, la visibilidad selectiva y la universalización de la violación sexual hacia las mujeres indígenas en Colombia: notas para la construcción de un campo de investigación", en Universitas Humanística,Vol. 84, No. 84, p.265-307.

Amnistía Internacional. (2004). Colombia Cuerpos marcados, crímenes silenciados. Violencia sexual contra las mujeres en el marco del conflicto armado. Madrid, España: Editorial Amnistía Internacional (EDAl).

Arroyo, Adriana \& Álvarez, María (2018): "Violencias cotidianas: perspectivas situadas desde las experiencias de niñas y mujeres en el municipio de Medellín, Colombia", en Sexualidad, Salud y Sociedad - Revista Latinoamericana, No. 29, p.123-146.

Benjumea, Adriana \& Loaiza, July (2018): Violencia sexual contra mujeres de Tumaco. Documentación y reflexión sobre los daños en mujeres racializadas (Corporación Humanas - Centro Regional de Derechos Humanos y Justicia de Género). Disponible en https://www.mpdl.org/sites/default/files/180530informe-tumaco.pdf

Boesten, Jelke (2010): "Analyzing Rape Regimes at the Interface of War and Peace in Peru", en The International Journal of Transitional Justice, Vol. 4, p.110-129.

Boesten, Jelke (2017): "Of exceptions and continuities: theory and methodology in research on conflict-related sexual violence", en International Feminist Journal of Politics, Vo. 19, No. 4, p.506-519.

Carrillo, Angela (2014): Buenaventura, Colombia: realidades brutales (Consejo Noruego para Refugiados-NRC). Disponible en: https://www.humanitarianresponse.info/sites/www.humanitarianresponse.info/fi les/documents/files/Buenaventura\%2C\%20colombia\%20realidades\%20Brutale s\%20Sept\%202014.pdf

Centro Nacional de Memoria Histórica [CNMH-Colombia]. (2017): La guerra inscrita en el cuerpo. Informe nacional de violencia sexual en el conflicto armado. Bogotá: Centro Nacional de Memoria Histórica. 
Céspedes-Báez, Lina-María (2010): "La violencia sexual en contra de las mujeres como estrategia de despojo de tierras en el conflicto armado colombiano", en Estudios Socio-Jurídicos, Vol. 12, No. 2, p.273-304. Disponible en: http://www.scielo.org.co/pdf/esju/v12n2/v12n2a9.pdf

Céspedes-Báez, Lina-María, Chaparro, Nina \& Estefan, Soraya (2014): "Metodologías en el estudio de la violencia sexual en el marco del conflicto armado interno", en Colombia Internacional, No. 80, p.19-56.

Chun, Suk \& Skjelsbæk, Inger (2010): "Sexual Violence in Armed Conflicts", en PRIO Policy Brief, No. 1. Oslo: PRIO. Disponible en: https://www.prio.org/utility/DownloadFile.ashx?id=235\&type=publicationfile

Cockburn, Cynthia (2004): "The Continuum of Violence: A Gender Perspective on War and Peace”, In Giles W. \& Hyndman J. (Eds.), Sites of Violence: Gender and Conflict Zones, p.24-44. Berkeley; Los Angeles; London: University of California Press.

Cruz, Alba, Calderón; Alejandra, Flórez, Sonia, \& Cardoso, Laura (2018): “Alabaos: El papel del cuidado en la sanación del dolor. Experiencia desde víctimas sobrevivientes de violencia sexual en el contexto colombiano" en Revista Guillermo de Ockham, Vol. 16, No. 2, p.115-124.

Defensoría del Pueblo [Colombia]. (2011): Violencia contra las mujeres en el Distrito de Buenaventura (Informe temático Defensoría delegada para la evaluación del riesgo de la población civil como consecuencia del conflicto armado Sistema de Alertas Temprana -SAT-). Disponible en: https://www.sdgfund.org/sites/default/files/Colombia VBG\%20Buenaventura.p df

Echeverri, Maria (2016): "Otredad racializada en la migración forzada de afrocolombianos a Antofagasta (Chile)", en Nómadas, No. 45, p.91-103. Disponible en: http://www.scielo.org.co/pdf/noma/n45/n45a07.pdf

Fiscó, Sonia (2005): “Atroces realidades: La violencia sexual contra la mujer en el conflicto armado colombiano", en Papel Político, No. 17, p.119-159. Disponible en: https://www.redalyc.org/pdf/777/77720407004.pdf

Foucault, Michel (2012): Historia de la sexualidad 1: la voluntad de saber. Buenos Aires: Siglo Veintiuno Editores.

Franco, Jean (2007): “Rape: A Weapon of War” en Social Text, Vol. 25, No. 2, 23-37.

Goldscheid, Julie (2020): "Gender Violence Against Afro-Colombian Women: Making the Promise of International Human Rights Law Real”, en Columbia Human Rights 
Revista Punto Género N.15 Junio de 2021

ISSN 0719-0417 / 25- 46

Law Review Online, 27 de mayo 2020, p.249-267. Disponible en: http://hrlr.law.columbia.edu/files/2020/05/10-Goldscheid Final.pdf

González Gil, Adriana (2012): "Mujeres, conflicto y desplazamiento forzoso: acción, resistencia y lucha por el reconocimiento y la inclusión", en ILEMATA, Vol. 4, No. 10, p.119-149. Disponible en: http://bibliotecadigital.udea.edu.co/bitstream/10495/6274/1/GonzalezAdriana 2 012 MujeresConflictoDesplazamientoforzosoLuchareconocimiento.pdf

Jimeno, Myriam (2007): "Lenguaje, subjetividad y experiencias de violencia”, en Antípoda, Revista de Antropología y Arqueología, No. 5, p.169-190.

Kreft, Anne-Kathrin (2020): "Civil society perspectives on sexual violence in conflict: patriarchy and war strategy in Colombia”, en International Affairs, Vol. 96, No. 2, p.457-478.

Lozano, Betty (2016): "Violencias contra las mujeres negras: Neo conquista y neo colonización de territorios y cuerpos en la región del Pacífico colombiano", en La manzana de la discordia, Vol. 11, No. 1, p.7-17.

Lucumí, Eva (2012): "Una mirada a las formas de subjetividad en mujeres víctimas de violencia sexual en el contexto de Buenaventura", en La manzana de la discordia, Vol. 7, No. 2, p.55-68.

Marciales, Carmen (2015): "Violencia sexual en el conflicto armado colombiano: racismo estructural y violencia basada en género", en Revista Via luris, No. 19, p.6990. Disponible en: https://www.redalyc.org/pdf/2739/273946366005.pdf

Meertens, Donny (1999): "Víctimas y sobrevivientes de la guerra: tres miradas de género", en Revista Estudios Interétnicos, Vol. 7. No. 11, p.28-38. Disponible en: http://c4.usac.edu.gt/revindex/articulos/editor9-r410 pi28 pfi38 ra576art.6.pdf

Meertens, Donny (2000): “El futuro nostálgico: desplazamiento, terror y género”, en Revista Colombiana De Antropología, No. 36, p.112-135. Recuperado de https://revistas.icanh.gov.co/index.php/rca/article/view/1300

Meger, Sara (2016): "The Fetishization of Sexual Violence in International Security", en International Studies Quarterly, Vol.60, No. 1, p.149-159.

Montoya, Sandra, Romero, María \& Jeréz, Lady (2013): "Mujer y desplazamiento de sí: sustratos socioculturales que soportan las redes de la violencia de género", en Revista Facultad Nacional Salud Pública, Vol. 31, No. 2, p.349-358. Disponible en: http://www.scielo.org.co/pdf/rfnsp/v31n3/v31n3a06.pdf 
Orduz, Frank (2015): "Victimización y violencia sexual en el conflicto armado en Colombia", en Subjetividad y Procesos Cognitivos, Vol. 19, No. 2, p.173-186. Disponible en: https://www.redalyc.org/pdf/3396/339643529009.pdf

Ozcurumez, Saime., Akyuz, Selin. \& Bradby, Hannah (2020): "The conceptualization problem in research and responses to sexual and gender-based violence in forced migration", en Journal of Gender Studies, p.1-13.

Pareja Amado, Antonio \& lañez Domínguez, Antonio (2014): "Violencia contra la mujer y desplazamiento forzado. Análisis de las estrategias de vida de jefas de hogar en Medellín", en Acta Sociológica, No. 65, p151-171.

Peñaranda, Bibiana \& Sulewski, David (2017): "Las mariposas de Buenaventura, Colombia: Sostienen la vida, construyen la paz", en Middle Atlantic Review of Latin American Studies, Vol. 1, No. 2, p.36-42.

Ruiz, Nubia (2011): "El desplazamiento forzado en Colombia: una revisión histórica y demográfica", en Estudios demográficos y urbano, Vol. 26, No. 1, p.141-177. Disponible en: https://www.redalyc.org/pdf/312/31224198005.pdf

Ruta Pacífica de las Mujeres (2013): La verdad de las mujeres víctimas del conflicto armado en Colombia. Disponible en: http://rutapacifica.org.co/documentos/versionresumidacomisiondeverdadymem oria.pdf

Salcedo, Edwin \& Paes-Machado, Eduardo (2019): "Victimización y desplazamiento forzado de mujeres en el conflicto armado colombiano", en MANA, Vol. 25, No. 1, p.95-125.

Segato, Rita (2003): Las estructuras elementales de la violencia. Ensayos sobre género entre la antropología, el psicoanálisis y los derechos humanos. Buenos Aires: Prometeo.

Segato, Rita (2016): La guerra contra las mujeres. Madrid: Traficantes de sueños.

Segura Escobar, Nora \& Meertens, Donny (1997): "Desarraigo, género y desplazamiento interno en Colombia", en Nueva Sociedad, No. 148, p.30-43. Disponible en: https://nuso.org/media/articles/downloads/2576 1.pdf

Skjelsbæk, Inger (2001): "Sexual Violence and War: Mapping Out a Complex Relationship", en European Journal of International Relations, Vol. 7, No. 2, p.211-237. 
Revista Punto Género N. 15 Junio de 2021

ISSN 0719-0417 / 25- 46

Skjelsbæk, Inger (2018): "Silence Breakers in War and Peace: Research on Gender and Violence with an Ethics of Engagement", en Social Politics: International Studies in Gender, State \& Society Vol. 25, No. 4, p.496-520.

Unidad para la atención y reparación integral a las víctimas [Colombia]. (2020). Registro único de víctimas. Disponible en: https://www.unidadvictimas.gov.co/es/registro-unico-de-victimas-ruv/37394

Urrego-Mendoza, Zulma (2007): "Las invisibles: Una lectura desde la salud pública sobre la violencia sexual contra niñas y mujeres colombianas en la actualidad", en Revista Colombiana de Obstetricia y Ginecología, Vol. 58, No. 1, p. 38-44. Disponible en: http://www.scielo.org.co/pdf/rcog/v58n1/v58n1a06.pdf

Wilches, Ivonne (2010): "Lo que hemos aprendido sobre la atención a mujeres víctimas de violencia sexual en el conflicto armado colombiano", en Revista de estudios Sociales, No. 36, p86-94. Disponible en: http://www.scielo.org.co/pdf/res/n36/n36a08.pdf

Wimmer, Andreas \& Glick Schiller, Nina (2003): Methodological Nationalism, the Social Sciences, and the Study of Migration: An Essay in Historical Epistemology", en International Migration Review, Vol. 37, No. 3, p.576-610.

Wirtz, Andrea, Pham, Kiemanh, Glass, Nancy, Loochkartt, Saskia, Kidane, Teemar, Cuspoca, Decssy, Rubenstein, Leonard, Singh, Sonal \& Vu, Alexander (2014): "Gender-based violence in conflict and displacement: qualitative findings from displaced women in Colombia", en Conflict and Health, Vol. 8, No. 10, p.1-14.

Wood, Elisabeth (2016): "La violencia sexual asociada al conflicto y las implicaciones políticas de investigaciones recientes", en Estudios Socio-Jurídicos, Vol. 18, No. 2, p.13-46.

Wood, Elisabeth (2019), "La violación como práctica de guerra: hacia una tipología de la violencia política”, en Revista Estudios Socio-jurídicos, Vol. 22. No. 1, p.67109. 